\title{
Loss of Essential Resources During the COVID-19 Pandemic Among Unsheltered and Unstably Housed Women
}

J Gen Intern Med 37(4):1017-9

DOI: $10.1007 / \mathrm{s} 11606-021-07288-1$

(C) The Author(s) under exclusive licence to Society of General Internal Medicine 2021

\section{INTRODUCTION}

Individuals living unsheltered or homeless are disproportionately vulnerable to severe forms of COVID-19. ${ }^{1}$ Contributing factors may include high rates of underlying health conditions and barriers to receiving care; however, the effects of the pandemic extend beyond physical health. We recently reported that social isolation and increased difficulties accessing care for chronic medical conditions during the pandemic were associated with poor mental health in unsheltered and unstably housed (UUH) women. ${ }^{2}$ What this means in the day-to-day lives of UUH women, who have specific social and mental health needs, is unclear but could inform future crisis-related emergency services. Studies prior to the pandemic suggest that many UUH women struggle to prioritize competing needs when managing their health, including transportation, time, and mon$\mathrm{ey},{ }^{3}$ issues which may be exacerbated by the pandemic.

We conducted a study to understand the impact of the COVID-19 pandemic on the day-to-day lives of UUH women. Our study is one of very few to directly query UUH women about their experiences during the pandemic.

\section{METHODS}

Between May and December 2020, we conducted phone surveys with women recruited from San Francisco homeless shelters, street encampments, free meal programs, and lowincome residential hotels. Participants were primarily recruited via flyers posted or distributed by service providers. An openended question asked participants to describe how they were personally impacted by the pandemic. Detailed notes and quotes were transcribed.

We used thematic analysis of survey data in accordance with steps outlined by Braun and Clarke. ${ }^{4}$ Two investigators independently read responses and generated codes from participants' recurring experiences and perceptions. Differences in coding were discussed until unity was reached. Codes were

Received August 23, 2021

Accepted November 17, 2021

Published online January 6, 2022 ultimately organized under themes (Table 1). Each theme represented a significant impact on participants' lives.

\section{RESULTS}

All 128 interviews included open-ended responses. We identified three main themes, represented in Figure 1 with illustrative quotes: exposure to significant lifestyle disruptions and losses, increased social isolation, and impaired access to essential resources. Many study participants reported increased feelings of marginalization in the wake of lifestyle disruptions. For others, social isolation and an inability to fulfill important social and familial roles coincided with the disruption of essential resources, leading to substantially increased difficulties meeting basic subsistence needs (Fig. 1).

While many in the general population have avoided or delayed seeking care during the pandemic to reduce health risks, ${ }^{5}$ UUH women faced additional barriers like increased costs or limited availability of transportation, closure of outpatient health services, and lack of internet access to participate in telemedicine. For example, participants described how

Table 1 Main Themes and Related Codes Associated with Unsheltered and Unstably Housed Women's Responses to the Open-Ended Question "How Have You Personally Been Impacted by the Pandemic?" $(N=128)$

\begin{tabular}{lll}
\hline \hline 1. Lifestyle disruptions and losses & $\mathbf{7 8}$ & $\mathbf{6 0 . 9 \%}$ \\
\hline $\begin{array}{l}\text { Disrupted lifestyle (unable to fulfill social/familial } \\
\text { roles or meet personal need) }\end{array}$ & 50 & $39.1 \%$ \\
$\quad$ Loss of routine & 26 & $20.3 \%$ \\
Financial hardship (impaired or reduced income, & 24 & $18.8 \%$ \\
increased expenses, unemployment) & 23 & $18.0 \%$ \\
Reduced locus of control & 18 & $14.1 \%$ \\
Impaired self-actualization & 11 & $8.6 \%$ \\
Elimination of in-person socialization & 10 & $7.8 \%$ \\
Loss of prior outlets for coping & 63 & $49.2 \%$ \\
2. Increased social isolation & 25 & $19.5 \%$ \\
Increased feeling of isolation & 17 & $13.3 \%$ \\
Fear of the SARS-CoV-2 virus & 17 & $13.2 \%$ \\
Increased self-imposed physical distance & 16 & $12.5 \%$ \\
Fear or distrust of others & 11 & $8.6 \%$ \\
Fear or concern for family members & 7 & $5.5 \%$ \\
Lack of empathy from, or frustration with, service & & \\
providers & 41 & $32.0 \%$ \\
3. Impaired access to essential resources & 22 & $17.2 \%$ \\
Disrupted healthcare service/reductions in access to & & \\
healthcare & 11 & $8.6 \%$ \\
Affected housing & 11 & $8.6 \%$ \\
Transportation disruption & 11 & $8.6 \%$ \\
Inability to access services & 11 & $8.6 \%$ \\
Food insecurity &
\end{tabular}

Responses listed are those provided by at least $5 \%$ of participants 


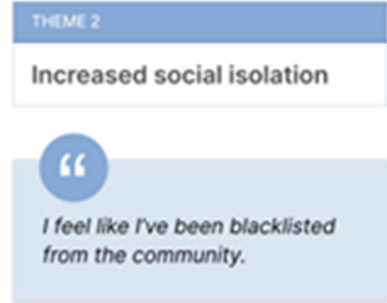

\section{(16)}

The housing doesn't feel supportive anymore. We feel forgotten.

\section{6}

I can't see my son in (his longterm care facility), and I worry about him. I need to see him. and look at his face, and look in his eyes. name 1

Lifestyle disruptions and losses

\section{(6)}

I can't go to the fibrary, and I used to go everyday [...] I was near food and a restroom and those are my main needs for the days.

\section{4}

I can't get medicine, I can't get food. I haven't seen my children since March, and theyre my support system.

Fig. 1 Thematic map of themes associated with unsheltered and unstably housed women's responses to the open-ended question "How have you personally been impacted by the pandemic?" $(N=128)$.

the elimination of bus routes led to difficulty accessing health clinics and food pantries and created additional financial burdens. One participant ordinarily attended mandatory biweekly urine drug tests to receive pain medication, but transportation vouchers were discontinued during the pandemic and increased transportation costs made it impossible for her to attend appointments. In addition, recently unsheltered participants, who were relocated to temporary housing, struggled to access food and medical care in new and unfamiliar settings.
Few participants specified positive effects of the pandemic and no positive themes were assigned.

\section{DISCUSSION}

During the COVID-19 pandemic, UUH women experienced significant lifestyle disruptions and losses, increased social 
isolation, and impaired access to essential resources. These experiences echo recent COVID-related isolation, loss, and lack of control in the general population. ${ }^{6}$ However, UUH individuals went into the pandemic with fewer resources and less social capital to lose. Safety measures that reduced services on which UUH women rely made the losses especially profound.

Measures addressing public health crises may have more impact, while also preserving the well-being of highly vulnerable populations, by maintaining accesses to essential resources (transportation, food pantries, and non-COVID health care). For example, creating new or at least temporary crisisaccess to technology like mobile phones and/or internet access could promote connections to resources, make telemedicine possible, and reduce isolation.

\section{Acknowledgements:}

Funding/Support: This research was supported by RGPO Emergency COVID-19 Research Seed Funding provided by the California HIV/ AIDS Research Program of the University of California (ROORG3097) and the National Institutes of Health (RO1 DA037012; RO1 DA049648; K24 DA039780).

Non-author Contribution: The authors thank study assistants Aron O'Donnell and Lizet Campos for their efforts with study development and data collection.

Aron O'Donnell, University of California, San Francisco, BS

Lizet Compos, University of California, San Francisco, BS

Access to Data and Data Analysis: Mr. Braun and Dr. Riley had full access to all the data in the study and take responsibility for the integrity of the data and the accuracy of the data analysis.

Originality of Content: All information and materials in this manuscript are original.

Carl Braun, $\mathrm{BS}^{1,2}$

Amanda N. Wade, MPH, BSN, RN ${ }^{1}$

Sheri D. Weiser, $M D, M P H^{1}$

Elise D. Riley, $P h D, M P H^{1}$

${ }^{1}$ Division of HIV, Infectious Diseases and Global Medicine, Department of Medicine, University of
California, San Francisco, San Francisco, CA, USA

${ }^{2}$ Albany Medical College, 43 New Scotland, MC1, Albany, NY 12208, USA

Corresponding Author: Carl Braun, BS; Albany Medical College, 43 New Scotland, MC1, Albany, NY 12208, USA (e-mail: braunc@amc.edu).

\section{Declarations:}

Conflict of interest: The authors declare that they do not have a conflict of interest.

Disclaimer: Funding organizations had no role in the design and conduct of the study; collection, management, analysis, and interpretation of the data; preparation, review, or approval of the manuscript; and decision to submit the manuscript for publication.

\section{REFERENCES}

1. Hsu HE, Ashe EM, Silverstein M, et al. Race/ethnicity, underlying medical conditions, homelessness, and hospitalization status of adult patients with COVID-19 at an urban safety-net medical center - Boston, Massachusetts, 2020. MMWR Morb Mortal Wkly Rep 2020;69(27):864-869.

2. Riley ED, Dilworth SE, Satre DD, et al. Factors associated with symptoms of depression and anxiety among women experiencing homelessness and unstable housing during the COVID-19 pandemic. JAMA Netw Open 2021;4(7):e2117035.

3. Allen J, Vottero B. Experiences of homeless women in accessing health care in community-based settings: a qualitative systematic review. JBI Evid Synth 2020;18(9):1970-2010.

4. Braun, V., \& Clarke, V. (2006). Using thematic analysis in psychology. Qual Res Psychol, 3(2), 77-101.

5. Fuller HR, Huseth-Zosel A. Older adults' loneliness in early COVID-19 social distancing: implications of rurality. J Gerontol B Psychol Sci Soc Sci. 2021:gbab053.

6. Imlach F, McKinlay E, Kennedy J, et al. Seeking healthcare during lockdown: challenges, opportunities and lessons for the future. Int $\mathrm{J}$ Health Policy Manag 202113.

Publisher's Note: Springer Nature remains neutral with regard to jurisdictional claims in published maps and institutional affiliations. 